\title{
Ultrasensitive broadband phototransistors based on perovskite/organic-semiconductor vertical heterojunctions
}

\begin{abstract}
Chao Xie ${ }^{1}$, Peng You ${ }^{1}$, Zhike Liu ${ }^{1}, \mathrm{Li} \mathrm{Li}^{2}$ and Feng Yan ${ }^{1}$
Organolead halide perovskites have emerged as the most promising materials for various optoelectronic devices, especially solar cells, because of their excellent optoelectronic properties. Here, we present the first report of low-voltage high-gain phototransistors based on perovskite/organic-semiconductor vertical heterojunctions, which show ultrahigh responsivities of $\sim 10^{9} \mathrm{~A} \mathrm{~W}^{-1}$ and specific detectivities of $\sim 10^{14}$ Jones in a broadband region from the ultraviolet to the near infrared. The high sensitivity of the devices is attributed to a pronounced photogating effect that is mainly due to the long carrier lifetimes and strong light absorption in the perovskite material. In addition, flexible perovskite photodetectors have been successfully prepared via a solution process and show high sensitivity as well as excellent flexibility and bending durability. The high performance and facile solution-based fabrication of the perovskite/organic-semiconductor phototransistors indicate their promise for potential application for ultrasensitive broadband photodetection.
\end{abstract}

Light: Science \& Applications (2017) 6, e17023; doi:10.1038/lsa.2017.23; published online 11 August 2017

Keywords: broadband; flexible; organic semiconductor; perovskite; photodetector

\section{INTRODUCTION}

Ultrasensitive photodetectors with a broadband spectral response from the ultraviolet (UV)-visible region to the near infrared (NIR) are highly desirable for a multitude of industrial and scientific applications ${ }^{1-5}$. The key strategy for realizing an ultrasensitive photodetector is to achieve a high gain, which means that one incident photon can induce many carriers to conduct current in the device. Conventional high-gain photodetectors, including avalanche photodiodes and photomultipliers, require strictly controlled and complicated fabrication procedures and are also expensive and bulky'. Moreover, these devices need to be operated at high voltages $(>100 \mathrm{~V})$, leading to high power consumption and inconvenience in many applications ${ }^{3-5}$. To date, various novel materials, including nanoparticles, nanorods, organic-semiconductors, graphene and other two-dimensional materials, have been exploited for the development of highly sensitive photodetectors ${ }^{3-5,7-17}$. To achieve high gain in a photodetector, one promising strategy is based on the photogating effect, which can cause photon-activated carriers to recirculate many times before recombining with opposite charges in the channel of a phototransistor $^{3,5}$. The photogating effect, similar to field-effect gating, refers to the modulation of the carrier density, and thus of the conductivity in the channel, by means of light illumination ${ }^{13}$. The sensitivity of a phototransistor relies on strong light absorption, efficient charge separation, long carrier lifetimes and high carrier mobilities in the channel. For example, graphene phototransistors modified with PbS colloidal quantum dots or $\mathrm{MoS}_{2}$ on the surfaces of the graphene have shown responsivities of up to $10^{8} \mathrm{~A} \mathrm{~W}^{-1}$ and gains of $\sim 10^{8}$ as a result of pronounced photogating effects ${ }^{13-15}$. However, the phototransistors with the highest previously reported gains cannot be prepared using facile solution processes. Therefore, it is necessary to explore solution-processable ultrasensitive phototransistors for lowcost and multifunctional applications.

Organolead halide perovskite materials have stimulated considerable research interest in relation to high-efficiency and low-cost perovskite solar cells ${ }^{18-20}$. Their excellent optoelectronic properties, including appropriate direct bandgaps ${ }^{21}$, high light absorption coefficient ${ }^{22}$ and long carrier transport lengths ${ }^{23-25}$, render them promising candidate materials for photovoltaics and other optoelectronic devices, including photodetectors, light-emitting diodes and semiconductor lasers ${ }^{26-30}$. Recently, organolead halide perovskites have been successfully used in various types of photodetectors, including photodiodes and phototransistors ${ }^{31-38}$; however, these devices have shown relatively low gain in comparison with phototransistors based on other functional materials. Moreover, previously reported perovskite photodetectors show a detectable wavelength region that is limited by the bandgap of the perovskite material and have not been used for sensing NIR light with wavelengths longer than $800 \mathrm{~nm}$.

Here, we report phototransistors based on perovskite/organicsemiconductor vertical heterojunctions prepared via a solution process. The devices contain double layers of $\mathrm{CH}_{3} \mathrm{NH}_{3} \mathrm{PbI}_{3-x} \mathrm{Cl}_{x}$

${ }^{1}$ Department of Applied Physics, The Hong Kong Polytechnic University, Hong Kong 999077, China and ${ }^{2}$ Institute of Textiles and Clothing, The Hong Kong Polytechnic University, Hong Kong 999077, China

Correspondence: F Yan, Email: apafyan@polyu.edu.hk

Received 11 August 2016; revised 8 February 2017; accepted 13 February 2017; accepted article preview online 22 February 2017 
a

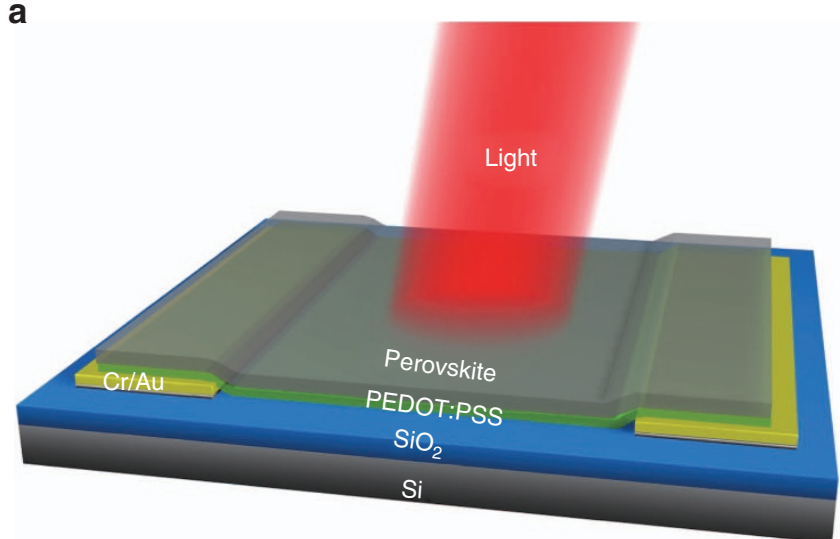

b

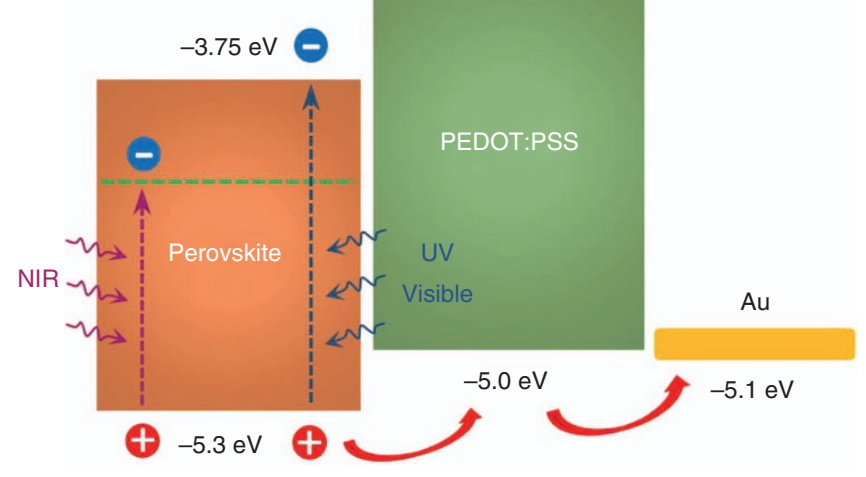

d

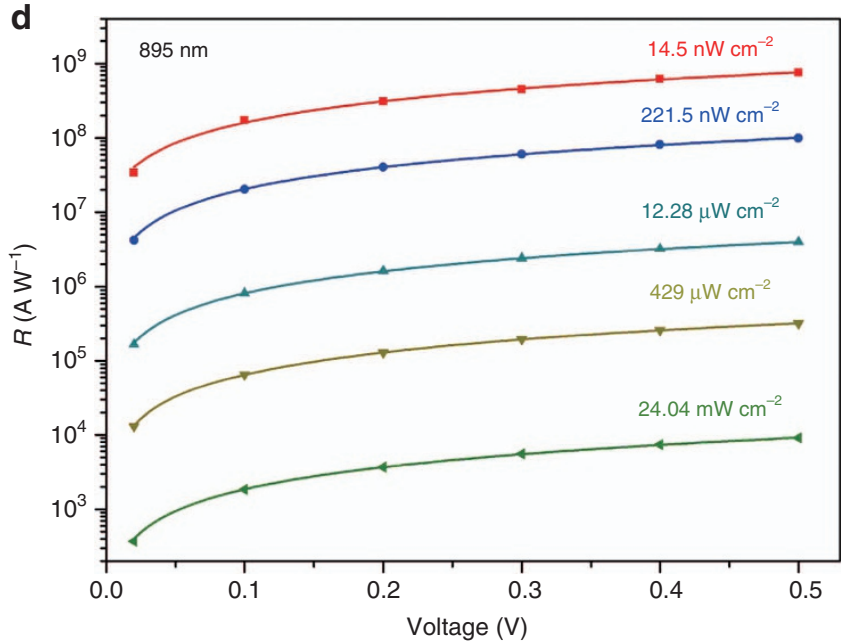

C

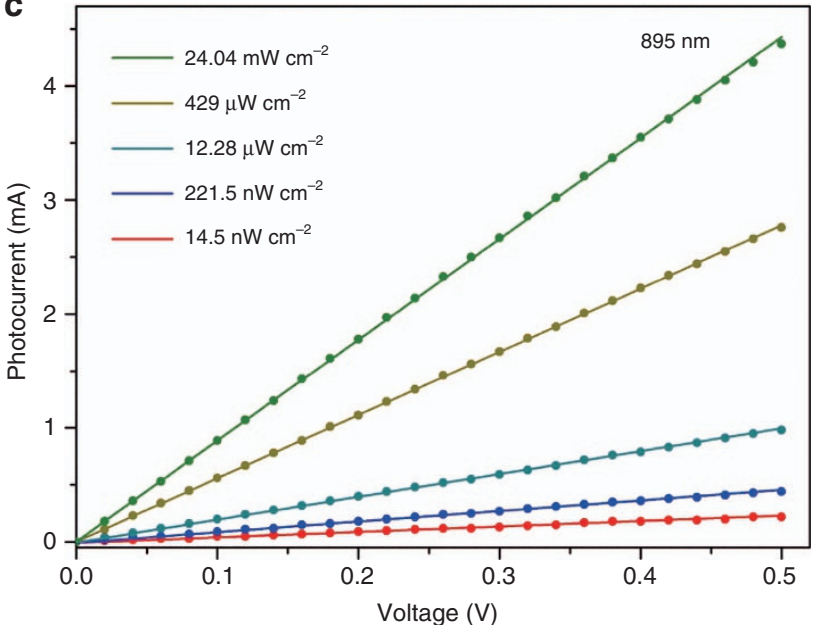



f



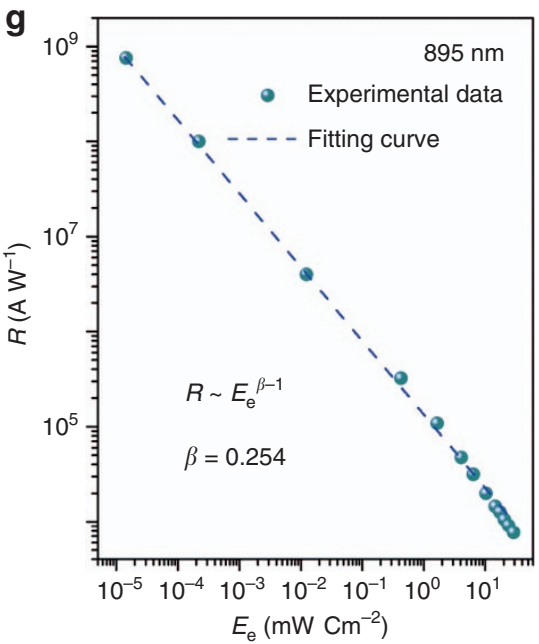

Figure 1 Design and performance of phototransistors based on a $\left.\mathrm{CH}_{3} \mathrm{NH}_{3} \mathrm{Pbl}_{3-x} \mathrm{Cl}\right|_{x} / \mathrm{PEDOT}$ :PSS heterojunction. (a) Schematic diagram of the device. (b) Energy band diagram and charge transfer behavior of the vertical heterojunction under illumination. (c) Photocurrent of a device as a function of the applied voltage as characterized under $895 \mathrm{~nm}$ light of various intensities. For each intensity, the device was illuminated for 5 min before the measurement. (d) Device responsivity as a function of the applied voltage for various light intensities. (e-g) Device responsivity as a function of the incident light intensity under light illumination at wavelengths of 370,598 and $895 \mathrm{~nm}$, respectively.

perovskite and an organic-semiconductor that form staggered heterojunctions. Although perovskite/organic heterojunctions have previously been successfully applied in photovoltaics and even in biological systems as artificial synapses ${ }^{39}$, they have never been used in phototransistors until now. We have found that phototransistors based on $\mathrm{CH}_{3} \mathrm{NH}_{3} \mathrm{PbI}_{3-x} \mathrm{Cl}_{x}$ and poly-(3,4-ethylenedioxythiophene): poly(styrenesulfonate) (PEDOT:PSS) exhibit a broadband photoresponse from the UV-visible region to the NIR region, with a responsivity and specific detectivity of higher than $10^{9} \mathrm{AW}^{-1}$ and $10^{14}$ Jones, respectively, at a low operating voltage of only $0.5 \mathrm{~V}$. This 
excellent performance is attributed to a significant photogating effect at the vertical heterojunction, mainly due to the long carrier lifetimes and strong light absorption in the perovskite layers.

\section{MATERIALS AND METHODS}

\section{Device fabrication}

A $\mathrm{SiO}_{2}(300 \mathrm{~nm}) / \mathrm{Si}$ or polyimide (PI) substrate was ultrasonically cleaned sequentially in alcohol, acetone and deionized water and dried under a stream of nitrogen gas. $\mathrm{Cr}(10 \mathrm{~nm}) / \mathrm{Au}(100 \mathrm{~nm})$ electrodes with a channel width $(W)$ and length $(L)$ of 1000 and $2 \mu \mathrm{m}$, respectively, were patterned via photolithography and magnetron sputtering. Then, a PEDOT:PSS film was deposited onto the substrate via the spin coating of a PEDOT:PSS aqueous solution (Clevios $\mathrm{PH}$ 500, Heraeus Precious Metals GmbH \& Co. KG, Leverkusen, Germany). The thickness of the PEDOT:PSS film was controlled by adjusting the solution concentration and the spin-coating speed. Afterward, the substrate was transferred into a glovebox filled with high-purity nitrogen gas and baked at $150^{\circ} \mathrm{C}$ for $1 \mathrm{~h}$.

Methylammonium iodide $\left(\mathrm{CH}_{3} \mathrm{NH}_{3} \mathrm{I}\right)$ and lead chloride $\left(\mathrm{PbCl}_{2}\right)$ (99.5 wt.\%) were dissolved in anhydrous N,N-dimethylformamide (DMF) (99.8 wt.\%) at a 3:1 molar ratio of $\mathrm{CH}_{3} \mathrm{NH}_{3} \mathrm{I}$ to $\mathrm{PbCl}_{2}$, and the solution was stirred at $45^{\circ} \mathrm{C}$ for $12 \mathrm{~h}$ inside a nitrogen-filled glovebox to produce a mixed halide perovskite precursor solution. Then, the precursor solution was spin coated onto the PEDOT:PSS film for $1 \mathrm{~min}$ to form a $\mathrm{CH}_{3} \mathrm{NH}_{3} \mathrm{PbI}_{3-x} \mathrm{Cl}_{x}$ perovskite film. The device was then annealed at $100{ }^{\circ} \mathrm{C}$ for $45 \mathrm{~min}$. The thickness of the perovskite film was controlled by adjusting the spin-coating speed while keeping the other conditions unchanged. In this work, perovskite films with approximate thicknesses of 180, 205, 250, 300 and $330 \mathrm{~nm}$ were obtained at spin-coating speeds of 6200, 4500, 3300, 2500 and 2000 r.p.m., respectively.

\section{Material characterization}

The morphology of the perovskite/PEDOT:PSS films was characterized using scanning electron microscopy (SEM, FEI Nova 450 Nano, FEI Company, Hillsboro, OR, USA). Transmittance and UV-vis absorption spectra were recorded using a Perkin Elmer (Waltham, MA, USA) Lambda 900 UV/VIS/NIR spectrometer. The PEDOT:PSS surface morphology was characterized by means of atomic force microscopy (AFM, Veeco NanoScope V, Bruker Corporation, Billerica, MA, USA). A surface chemical analysis of the PEDOT:PSS was conducted using an X-ray photoelectron spectrometer (VG ESCALAB 220i-XL, Thermo Fisher Scientific, Waltham, MA, USA).

\section{Device characterization}

Electrical and optoelectrical measurements were performed using a semiconductor parameter analyzer (Agilent 4156 C, Santa Clara, CA, USA) under light illumination at various intensities in a glovebox. The light sources were light-emitting diodes with wavelengths of 370, 598 and $895 \mathrm{~nm}$. The flexible devices were measured both before and after bending tests. In the bending tests, each sample was pressed against a small glass bottle with a radius of $7 \mathrm{~mm}$ up to 300 times. Spectral response tests were performed using a standard quantum efficiency measurement system equipped with a xenon lamp (Oriel 66902, $300 \mathrm{~W}$, Newport Corporation, Irvine, CA, USA), a monochromator (Newport 66902, Irvine, CA, USA), a Si detector (Oriel 76175-71580, Newport Corporation), and a dual-channel power meter (Newport 2931-C). Neutral density filters were used to attain light with a constant intensity, and a source meter (Keithley 2420, Cleveland, $\mathrm{OH}$, USA) was used to collect the electrical signals. The devices were wire- bonded and encapsulated using glass caps and epoxy in the glovebox before the spectral response tests in air.

\section{RESULTS AND DISCUSSION}

Figure 1a presents a schematic diagram of a perovskite photodetector consisting of $\mathrm{CH}_{3} \mathrm{NH}_{3} \mathrm{PbI}_{3-x} \mathrm{Cl}_{x}$ perovskite and PEDOT:PSS films on a $\mathrm{SiO}_{2} / \mathrm{Si}$ substrate. $\mathrm{Cr} / \mathrm{Au}$ electrodes were patterned on the substrate by means of photolithography and magnetron sputtering, followed by the sequential coating of PEDOT:PSS and $\mathrm{CH}_{3} \mathrm{NH}_{3} \mathrm{PbI}_{3-x} \mathrm{Cl}_{x}$ perovskite layers. The surface morphology of the perovskite film on the PEDOT: PSS was observed under SEM (see the Supplementary Information and Supplementary Fig. S1). We found that the perovskite layer exhibited reasonably full coverage on the PEDOT:PSS layer, with only a few pinholes, and cross-sectional SEM imaging showed the film to have a double-layer stacked structure.

Figure $1 \mathrm{~b}$ shows the band structure of the device ${ }^{29}$, in which a staggered heterojunction is formed between the $\mathrm{CH}_{3} \mathrm{NH}_{3} \mathrm{PbI}_{3-x} \mathrm{Cl}_{x}$ perovskite and the PEDOT:PSS. Under light illumination, excitons or electron-hole pairs are generated in the perovskite film, depending on the film morphology, as reported by Grancini et al. ${ }^{40}$. Moreover, excitons can easily dissociate into electrons and holes at the perovskite/ PEDOT:PSS interface or in the perovskite film because of the lowexciton binding energy ${ }^{41}$. Holes tend to diffuse into the PEDOT:PSS layer because of the decreased energy, whereas electrons must accumulate in the perovskite layer since the conduction band level of the perovskite is lower than the lowest unoccupied molecular orbital level of the PEDOT:PSS. Thus, the channel current of the device can be attributed to the electrons and holes in the perovskite and PEDOT: PSS layers, respectively. We found that the electron mobility in the perovskite layer is only $\sim 1.7 \times 10^{-4} \mathrm{~cm}^{2} \mathrm{~V}^{-1} \mathrm{~s}^{-1}$ (Supplementary Information and Supplementary Fig. S2), whereas the hole mobility in the PEDOT:PSS layer is estimated to be $\sim 5.6 \mathrm{~cm}^{2} \mathrm{~V}^{-1} \mathrm{~s}^{-1}$ (Supplementary Information, Parts 1 and 2). Therefore, the channel current is mainly due to the hole transport in the PEDOT:PSS layer, and the current across the perovskite layer is negligible.

The current-voltage $(I-V)$ curves between the two Au electrodes of the perovskite photodetectors were first measured in the dark. The devices exhibited linear $I-V$ curves, indicating good ohmic contacts between the perovskite/PEDOT:PSS bilayer films and the Au electrodes. Then, the perovskite photodetectors were characterized under light illumination at wavelengths from the UV-visible region to the NIR region. Figure $1 \mathrm{c}$ shows the photocurrent $\left(I_{\mathrm{ph}}=I_{\text {light }}-I_{\text {dark }}\right)$ of a device as a function of the applied voltage between the two Au electrodes under illumination with NIR light (wavelength: $\sim 895 \mathrm{~nm}$ ) at various intensities. The photocurrents of the same device under UV $(\sim 370 \mathrm{~nm})$ and visible $(\sim 598 \mathrm{~nm})$ light are shown in the Supplementary Information (Supplementary Fig. S3). The photocurrent was found to depend almost linearly on the applied voltage, with a maximum value of $\sim 4 \mathrm{~mA}$ at an applied voltage of only $0.5 \mathrm{~V}$. To confirm that the photocurrents reported above were not due solely to either the perovskite or the PEDOT:PSS film, control experiments were performed on devices prepared with the individual films. We found that the photocurrent of a transistor based on $\mathrm{CH}_{3} \mathrm{NH}_{3} \mathrm{PbI}_{3-x} \mathrm{Cl}_{x}$ perovskite alone was $\sim 10 \mathrm{nA}$ at a bias voltage of $0.5 \mathrm{~V}$ under light illumination at the same intensity (see the Supplementary Information, Supplementary Fig. S4). Another control device, based on pure PEDOT:PSS, exhibited a negligible current response to light illumination. The photocurrents $(\sim 1 \mu \mathrm{A})$ were three orders of magnitude lower than the values observed in the perovskite/ PEDOT:PSS phototransistors under the same light intensity from the UV-visible to the NIR region (Supplementary Fig. S5). Therefore, the 
increase in the channel current of a perovskite/PEDOT:PSS phototransistor under light illumination can be attributed to a photogating effect, and the photocurrent $I_{\mathrm{ph}}$ is given by

$$
I_{\mathrm{ph}}=I_{\text {light }}-I_{\text {dark }}=\frac{W}{L} q \Delta p \mu V,
$$

where $I_{\text {light }}$ and $I_{\text {dark }}$ are the currents in the device under light illumination and in the dark, respectively; $W$ and $L$ are the channel width and length of the device, respectively; $q$ is the elementary charge; $\Delta p$ is the increase in the density of holes per unit area in the PEDOT: PSS layer in the channel; $\mu$ is the hole mobility in the PEDOT:PSS layer; and $V$ is the voltage applied to the two Au electrodes.

The responsivity $(R)$, which is an essential figure of merit for a photodetector, is given $b^{6}$

$$
R\left(\mathrm{AW}^{-1}\right)=\frac{I_{\mathrm{ph}}}{E_{e} W L},
$$

where $E_{e}$ is the light intensity. Figure $1 \mathrm{~d}$ shows the device responsivity as a function of the applied voltage for various light intensities (wavelength: $895 \mathrm{~nm}$ ), exhibiting a maximum value of $7.6 \times 10^{8} \mathrm{~A} \mathrm{~W}^{-1}$. Figures $1 \mathrm{e}-1 \mathrm{~g}$ show the responsivities to wavelengths of 370,598 and $895 \mathrm{~nm}$, respectively. The responsivity increased as the light intensity decreased, following a relationship of the form $R \propto E_{e}^{\beta-1}$, as previously observed in many other phototransistors ${ }^{3,14}$. Notably, the highest responsivity at low light intensity was $1.91 \times 10^{9} \mathrm{~A} \mathrm{~W}^{-1}$ (wavelength: $\sim 598 \mathrm{~nm}$ ), which is much higher than the corresponding responsivities of previously reported perovskite photodetectors and other state-of-the-art photodetectors ${ }^{6,13-15,31-38}$. Moreover, the gain $(G)$ of a phototransistor is given by ${ }^{42}$

$$
G=\frac{I_{\mathrm{ph}} h c}{q E_{e} W L \lambda}=\frac{R h c}{q \lambda}
$$

where $c$ is the speed of light, $\lambda$ is the wavelength of the incident light and $h$ is Planck's constant. Thus, the gain corresponding to the maximum responsivity is estimated to be $4.0 \times 10^{9}$.

In addition to the responsivity, the specific detectivity $\left(D^{*}\right)$ is another key parameter of a photodetector and is given by $\mathrm{y}^{6,42-44}$

$$
\begin{gathered}
D^{*}=\frac{(A B)^{1 / 2}}{N E P} \\
N E P=\frac{{\overline{i_{n}^{2}}}^{1 / 2}}{R}
\end{gathered}
$$

where $A$ is the effective area of the photodetector, $B$ is the bandwidth, NEP is the noise equivalent power, $\bar{i}_{m}^{-1 / 2}$ is the root mean square value of the noise current and $R$ is the responsivity of the device. The noise level per unit bandwidth $(1 \mathrm{~Hz})$ of the perovskite photodetectors was measured to be $\sim 60 \mathrm{nA} \mathrm{Hz}^{-1 / 2}$ (see the Supplementary Information, Part 3 and Supplementary Fig. S10). Therefore, the specific detectivities $\left(D^{*}\right)$ of a device at an applied voltage of $0.5 \mathrm{~V}$ and at wavelengths of 598 and $895 \mathrm{~nm}$ were $1.4 \times 10^{14}$ and $5.6 \times 10^{13}$ Jones $\left(\mathrm{cm} \mathrm{Hz}^{1 / 2} \mathrm{~W}^{-1}\right)$, respectively, far superior to those of traditional Si photodetectors ${ }^{6}$.

Figure 2a shows a schematic diagram of a perovskite/PEDOT:PSS phototransistor under light illumination, in which electrons and holes have accumulated in the perovskite and PEDOT:PSS layers, respectively. The density of photocarriers (that is, photo-generated holes) in the PEDOT:PSS layer can be calculated based on the photocurrent. If the hole mobility under light illumination is assumed to be constant, then the density of photocarriers in the PEDOT:PSS layer is given by the following equation ${ }^{6}$ :

$$
\Delta n=\frac{L}{W q \mu V} I_{\mathrm{ph}}
$$

where $\mu$ is the hole mobility in PEDOT:PSS. As shown in Figure $2 \mathrm{~b}$, the maximum hole density that can be achieved is $\sim 2 \times 10^{13} \mathrm{~cm}^{-2}$, which is one order of magnitude higher than that typically induced in a field-effect transistor ${ }^{6}$. Therefore, an extremely strong photogating effect is observed in the devices under light illumination.

We further characterized the spectral response of the perovskite photodetectors as shown in Figure 2c. The normalized spectral responsivity of a device at a given light intensity exhibits a sharp rising edge near a wavelength of $800 \mathrm{~nm}$, corresponding to the bandedge absorption of the $\mathrm{CH}_{3} \mathrm{NH}_{3} \mathrm{PbI}_{3-x} \mathrm{Cl}_{x}$ perovskite $(\sim 1.55 \mathrm{eV})$, as shown in the same figure. Although the responsivity is rather low at wavelengths longer than $800 \mathrm{~nm}$, it can be as high as $7.6 \times 10^{8} \mathrm{~A} \mathrm{~W}^{-1}$ at $895 \mathrm{~nm}$, corresponding to a gain of $1.1 \times 10^{9}$. Notably, the spectral response of the phototransistors is different from the external quantum efficiency of a perovskite solar cell, which shows a sharp decrease to 0 for wavelengths longer than $800 \mathrm{~nm}$ (see the Supplementary Information and Supplementary Fig. S11) ${ }^{45}$. To better understand this effect, the light absorption of a pure perovskite film was characterized from the UV to the NIR region. As shown in Figure 2c, a perovskite film can absorb light at wavelengths of up to $1.9 \mu \mathrm{m}$ because of the existence of traps in the film ${ }^{46}$. Therefore, the photoresponse of the devices at wavelengths longer than $800 \mathrm{~nm}$ can be attributed to the excitation of carriers from the valence band to the trap states within the perovskite bandgap, as shown in Figure 1b. In such a phototransistor, the photo-generated electrons are not necessarily mobile in the perovskite film, and thus, an ultrahigh responsivity in the NIR region can be achieved, unlike in the case of a solar cellbased photodetector ${ }^{33,34}$.

The photoresponse of a device also depends on the thicknesses of the perovskite and PEDOT:PSS layers, as shown in Figures $2 \mathrm{c}$ and $2 \mathrm{~d}$. The responsivity increases with increasing perovskite thickness because of the enhanced light absorption and tends to saturate when the perovskite thickness is larger than $300 \mathrm{~nm}$. Similarly, the responsivity increases with increasing PEDOT:PSS thickness and reaches a maximum when this thickness is $\sim 43 \mathrm{~nm}$. Therefore, the thicknesses of the perovskite and PEDOT:PSS layers were optimally chosen to be $300 \mathrm{~nm}$ and $43 \mathrm{~nm}$, respectively, in our experiments.

The time-dependent photoresponse of the perovskite photodetectors was studied by periodically turning the light illumination on and off. As shown in Figure 3a, the photocurrent of a device increases rapidly during the initial stage (within the first few seconds) of illumination and then increases slowly for several minutes; this behavior can be fitted using a double exponential function with two relaxation times

$$
I_{\mathrm{ph}}=A_{1}\left(1-\exp \left(-\frac{t}{\tau_{1}}\right)\right)+A_{2}\left(1-\exp \left(-\frac{t}{\tau_{2}}\right)\right)
$$

where $\tau_{1}$ and $\tau_{2}$ are two time constants and $A_{1}$ and $A_{2}$ are magnitudes. As shown in Figure $3 \mathrm{~b}$, the time constants $\tau_{1}$ and $\tau_{2}$ for the fitted curve are 4.5 and $57.5 \mathrm{~s}$, respectively. We note that these response times are relatively long compared with previously reported perovskite photodetectors ${ }^{31,32,35,36}$, which is not strange since slow responses are typically expected in phototransistors with ultrahigh gains ${ }^{13-15,47}$. In our devices, the photoresponse is associated with the accumulation processes of holes and electrons in the PEDOT:PSS and perovskite layers, respectively. During the initial stage, holes diffuse from the perovskite to the PEDOT:PSS layer, and thus, the channel current 
a

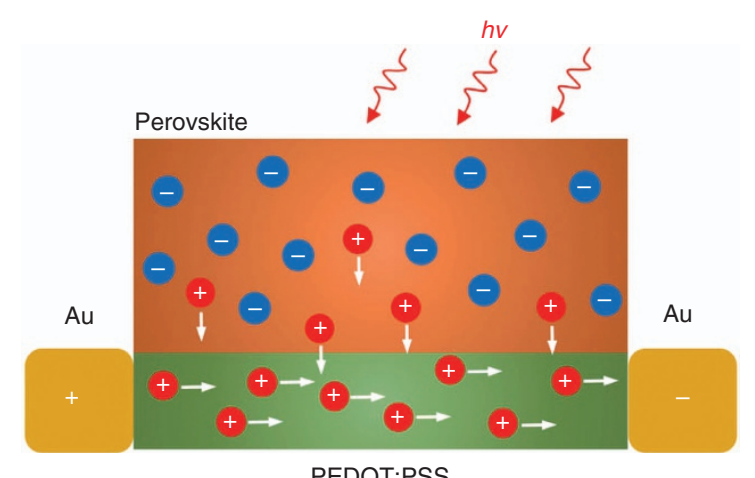

PEDOT:PSS

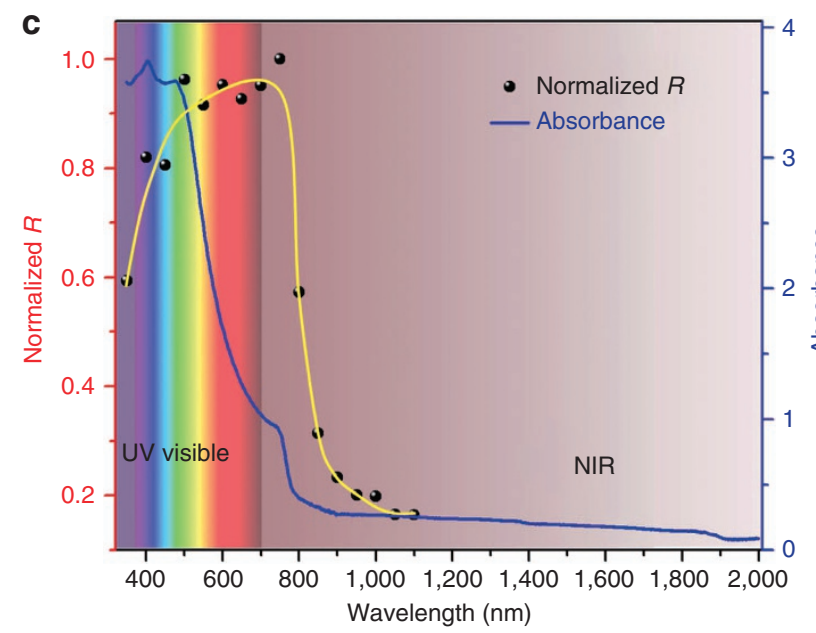

b
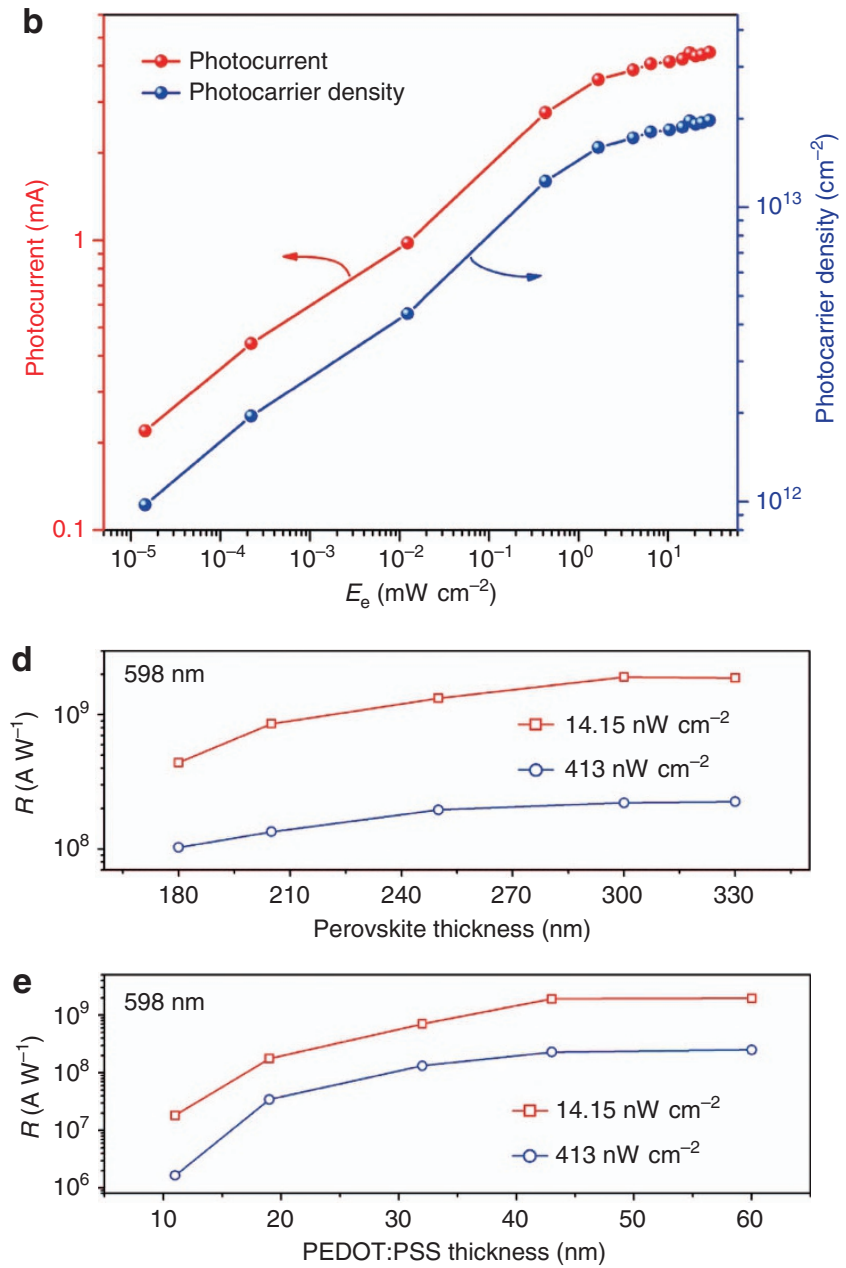

Figure 2 Photoresponse of $\mathrm{CH}_{3} \mathrm{NH}_{3} \mathrm{Pbl}_{3-x} \mathrm{Cl}_{x} / \mathrm{PEDOT}$ :PSS phototransistors. (a) Schematic diagram of a perovskite/PEDOT:PSS phototransistor under light illumination. (b) Plot of the photocurrent and photocarrier density of a phototransistor as functions of the light intensity. Light wavelength: $895 \mathrm{~nm}$. (c) Normalized spectral responsivity of a device (wavelengths: from 350 to $1100 \mathrm{~nm}$ ) and absorbance spectrum of a $\mathrm{CH}_{3} \mathrm{NH}_{3} \mathrm{Pbl}_{3-x} \mathrm{Cl}_{x}$ perovskite film on a glass substrate (wavelengths: from 350 to $2000 \mathrm{~nm}$ ). The black dots represent the experimental data, and the yellow line is a guide for the eye. ( $\mathbf{d}$ and $\mathbf{e}$ ) Responsivities of the phototransistors as functions of the perovskite thickness and PEDOT:PSS thickness, respectively. Light wavelength: $598 \mathrm{~nm}$.

rapidly increases with time. As the hole density in the channel increases, more holes will recombine with electrons at the perovskite/PEDOT:PSS interface. Moreover, a reverse electric field induced by the increased number of separated charge carriers will depress the injection rate of holes. Consequently, the increase in channel current subsequently slows under continued illumination for the above reasons. Only once the recombination rate is equal to the rate of injection of holes from the perovskite to the PEDOT:PSS can the device show a stable photocurrent. At the same time, holes will transfer and recirculate many times in the PEDOT:PSS channel throughout their long carrier lifetimes, which explains the ultrahigh gain achieved in our phototransistors.

According to Equation (3), the gain of a device can be calculated based on its time-dependent photocurrent. Figure $3 \mathrm{c}$ shows the gain of a device as a function of illumination time for two different light intensities. The gain of the device increased over time and saturated at values of $1.2 \times 10^{9}$ and $2.9 \times 10^{6}$ for light intensities of 14.15 and $76 \mu \mathrm{W} \mathrm{cm}{ }^{-2}$, respectively. Thus, the gain decreases with increasing light intensity, which is consistent with the decreased responsivity with increasing light intensity shown in Figure 1.
Since the photoresponse of our devices is due to the accumulation of electrons in the perovskite layer, it is reasonable to consider that the response speed of a device is closely related to the properties of its perovskite layer. We measured a phototransistor with a thinner perovskite layer (thickness: $\sim 180 \mathrm{~nm}$ ) prepared under the same annealing conditions as the other devices (see the Supplementary Information, Part 4 and Supplementary Fig. S12). Although the responsivity of the device was lower, which is consistent with the data shown in Figure 2d, the response speed was dramatically improved, indicating that a thinner perovskite layer can lead to a shorter response time. During the charge accumulation process in the perovskite/PEDOT:PSS heterojunction, electrons will fill the trap states from deeper to shallower levels and will then fill the conduction band in the perovskite. The deep trap states can be filled throughout the entire perovskite layer because of their low-energy levels, whereas the shallow trap states and conduction band will be filled only close to the heterojunction. Consequently, as the film thickness decreases, fewer deep trap states will be involved in the charge accumulation process. Since the capture cross section of deep trap states is typically smaller than that of shallow trap states ${ }^{6}$, a thinner perovskite layer with fewer 

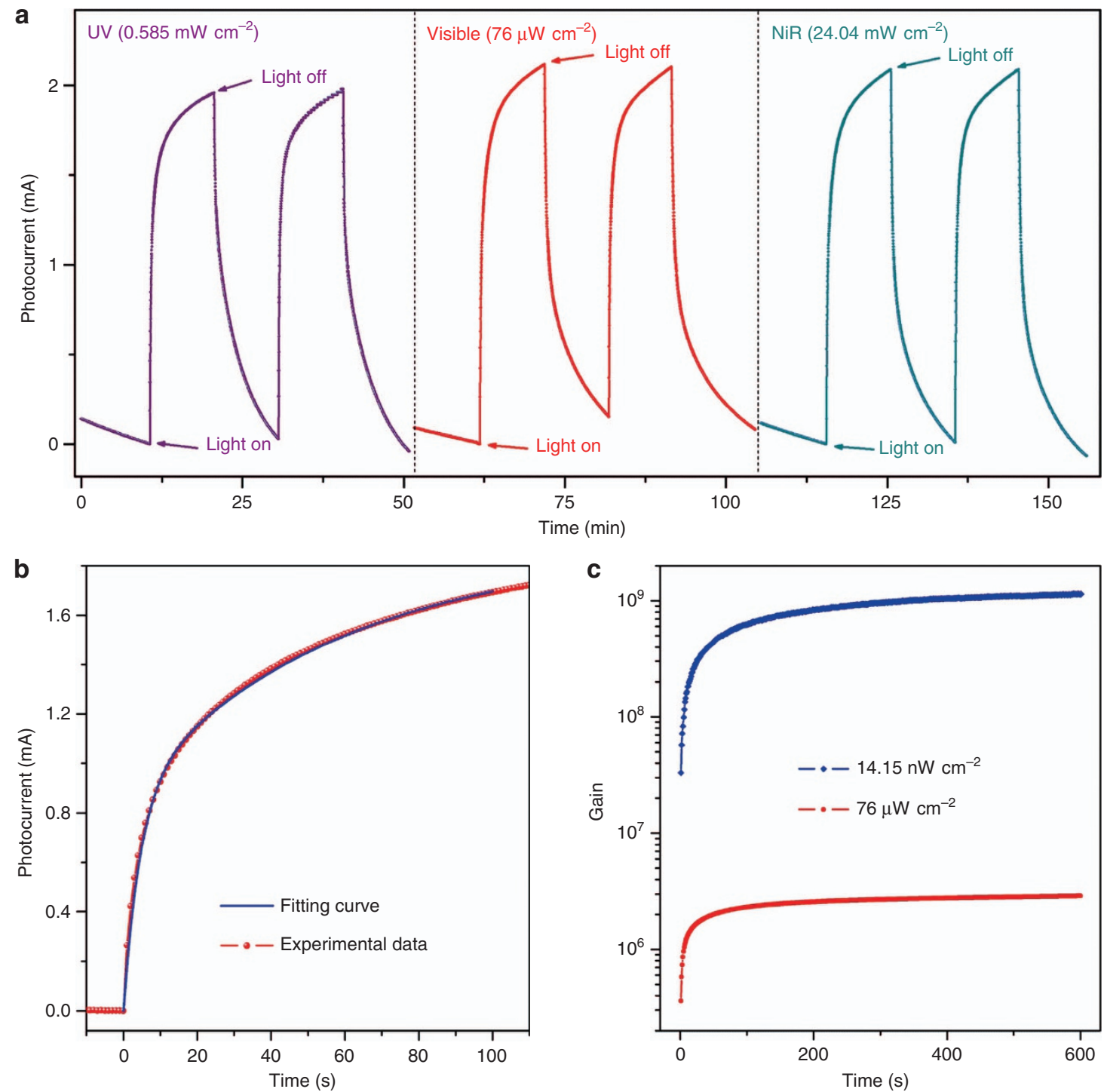

Figure 3 Transient properties of a $\mathrm{CH}_{3} \mathrm{NH}_{3} \mathrm{Pbl}_{3-x} \mathrm{Cl} /$ PEDOT:PSS phototransistor. (a) Time-dependent photoresponse of the photodetector to periodic on/off illumination at three different wavelengths of 370,598 and $895 \mathrm{~nm}$. (b) Enlarged view of the rising edge of the time-dependent photoresponse fitted with a double exponential function. Light wavelength: $598 \mathrm{~nm}$. (c) Gain of the phototransistor as a function of time under light illumination (wavelength: $598 \mathrm{~nm}$ ) at intensities of $14.15 \mathrm{nW} \mathrm{cm}-2$ and $76 \mu \mathrm{W} \mathrm{cm}^{-2}$.

deep trap states can result in a phototransistor with a faster response speed.

Next, we investigated the properties of the perovskite and organicsemiconductor layers that might influence the device responsivity. Although the perovskite material shows strong light absorption and can generate electron-hole pairs with high quantum efficiency, some holes recombine with electrons in the perovskite and are unable to be injected into the PEDOT:PSS layer, especially when a high density of electrons accumulates in the perovskite layer. Therefore, the responsivity and gain of a device strongly depend on the lifetime of holes in the perovskite, which is related to two factors. First, the density of electrons in the perovskite layer increases with increasing light intensity, leading to a decrease in the hole lifetime in the perovskite and, consequently, a decrease in device responsivity. Second, the hole lifetime in $\mathrm{CH}_{3} \mathrm{NH}_{3} \mathrm{PbI}_{3-x} \mathrm{Cl}_{x}$ perovskite has been found to be longer than those in many other semiconductors because of the low density of recombination centers in the perovskite ${ }^{48}$. To further confirm this understanding, we tested phototransistors based on $\mathrm{CH}_{3} \mathrm{NH}_{3} \mathrm{PbI}_{3} /$ PEDOT:PSS heterojunctions (see the Supplementary Information, Part 5 and Supplementary Fig. S13) and found that the maximum responsivity was only $\sim 10^{6} \mathrm{~A} \mathrm{~W}^{-1}$ at the lowest light intensity. It has been reported that the hole lifetime in a $\mathrm{CH}_{3} \mathrm{NH}_{3} \mathrm{PbI}_{3}$ film is much shorter than that in a $\mathrm{CH}_{3} \mathrm{NH}_{3} \mathrm{PbI}_{3-x} \mathrm{Cl}_{x}$ film under the same illumination conditions $s^{23,49}$. Therefore, it is reasonable to find that a phototransistor based on $\mathrm{CH}_{3} \mathrm{NH}_{3} \mathrm{PbI}_{3} / \mathrm{PEDOT}$ :PSS shows a much lower responsivity than a device based on $\mathrm{CH}_{3} \mathrm{NH}_{3} \mathrm{PbI}_{3-x} \mathrm{Cl}_{x} / \mathrm{PEDOT}$ : PSS. This experiment clearly indicates that the ultrahigh responsivity and gain of our phototransistors are due to the long lifetime and diffusion length of holes in the $\mathrm{CH}_{3} \mathrm{NH}_{3} \mathrm{PbI}_{3-x} \mathrm{Cl}_{x}$ perovskite films.

To better understand the photogating effect of the perovskite/ organic-semiconductor heterojunction, we prepared devices based on another organic-semiconductor, poly(3-hexylthiophene) (P3HT), and $\mathrm{CH}_{3} \mathrm{NH}_{3} \mathrm{PbI}_{3-x} \mathrm{Cl}_{x}$. P3HT is a typical organic-semiconductor with a carrier mobility of up to $0.1 \mathrm{~cm}^{2} \mathrm{~V}^{-1} \mathrm{~s}^{-1}$ (Ref. 50). Under light 
a

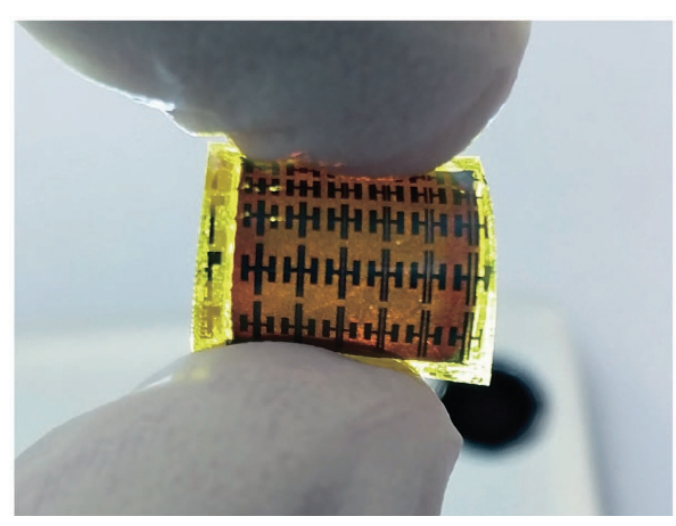

C

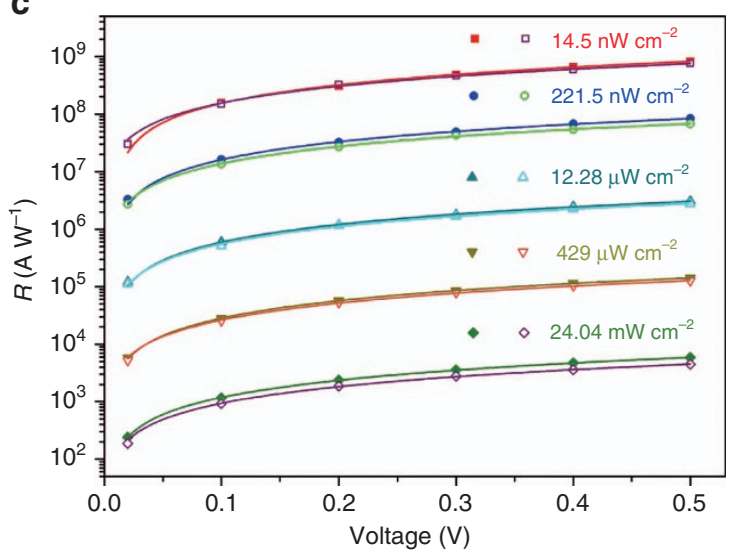

b

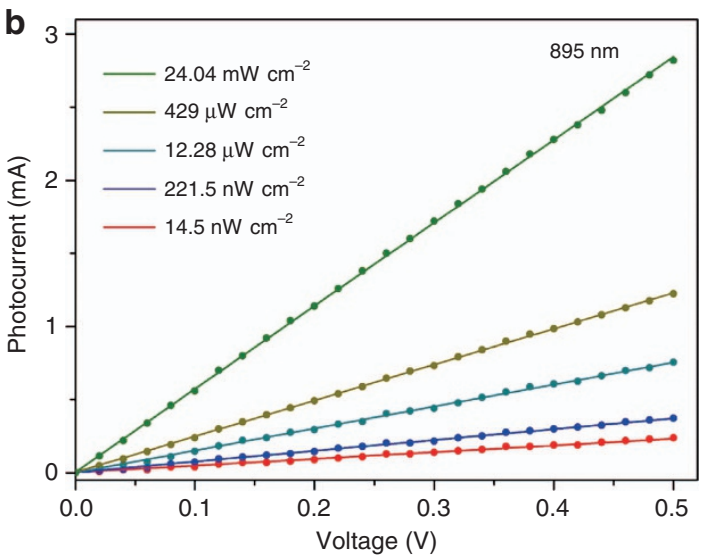

d
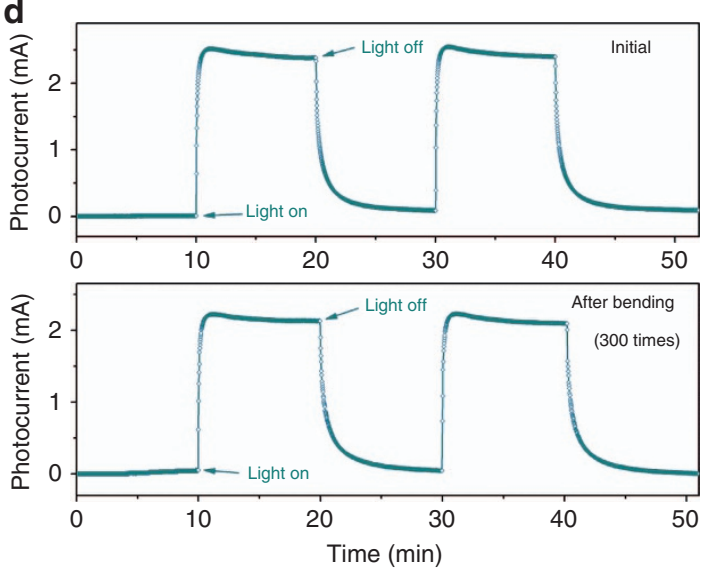

Figure 4 Performance of a flexible $\mathrm{CH}_{3} \mathrm{NH}_{3} \mathrm{Pbl}_{3-x} \mathrm{Cl} \mathrm{l}_{x}$ PEDOT:PSS phototransistor. (a) Photograph of the flexible phototransistor. (b) Photocurrent of the device as a function of the applied voltage under various NIR light intensities (wavelength: $895 \mathrm{~nm}$ ). For each intensity, the device was illuminated for 5 min before the measurement. (c) Responsivity of the flexible phototransistor before (solid dots) and after (hollow dots) a bending test. (d) Time-dependent photoresponse of the device to periodic on/off illumination before and after the bending test. Light wavelength: $895 \mathrm{~nm}$.

illumination, the transfer curve of such a transistor shifts toward higher gate voltages because of the injection of holes into the P3HT layer (see the Supplementary Information and Supplementary Fig. S14). The increase in hole density induced by light illumination is given by $\Delta n=\frac{C_{i} \Delta V}{q}$, where $C_{i}$ is the gate capacitance and $\Delta V$ is the light-induced shift in the gate voltage. The maximum shift is $\sim 20 \mathrm{~V}$, corresponding to the increase in the hole density in the channel to $4 \times 10^{12} \mathrm{~cm}^{-2}$, which is much lower than the value $\left(2 \times 10^{13} \mathrm{~cm}^{-2}\right)$ observed for devices based on $\mathrm{CH}_{3} \mathrm{NH}_{3} \mathrm{PbI}_{3-x} \mathrm{Cl}_{x} / \mathrm{PEDOT}$ :PSS. More importantly, the responsivity (see the Supplementary Information and Supplementary Fig. S14 and S15) is several orders of magnitude lower than that of a device based on $\mathrm{CH}_{3} \mathrm{NH}_{3} \mathrm{PbI}_{3-x} \mathrm{Cl}_{x} / \mathrm{PEDOT}$ :PSS for two reasons. First, the hole mobility of P3HT is only $2.7 \times 10^{-2} \mathrm{~cm}^{2} \mathrm{~V}^{-1} \mathrm{~s}^{-1}$, which is two orders of magnitude lower than that of PEDOT:PSS. Second, the maximum density of accumulated carriers in a heterojunction is related to the status of the energy level alignment on both sides. Under light illumination, a $\mathrm{CH}_{3} \mathrm{NH}_{3} \mathrm{PbI}_{3-x} \mathrm{Cl}_{x} / \mathrm{P} 3 \mathrm{HT}$ heterojunction has band bending on both sides, whereas a $\mathrm{CH}_{3} \mathrm{NH}_{3} \mathrm{PbI}_{3-x} \mathrm{Cl}_{x} / \mathrm{PEDOT}$ :PSS heterojunction has band bending only on the perovskite side because the PEDOT:PSS is highly doped and the Fermi level inside it is pinned by the dopant (see the Supplementary Information and Supplementary Fig. S16). Consequently, a much higher density of electrons can accumulate in the $\mathrm{CH}_{3} \mathrm{NH}_{3} \mathrm{PbI}_{3-x} \mathrm{Cl}_{x} / \mathrm{PEDOT}$ :PSS heterojunction. Meanwhile, the strong photogating effect can be attributed to the high relative dielectric constant $(\sim 60)$ of the perovskite material ${ }^{41}$, which is much higher than that of organic-semiconductors $(\sim 3)$ or $\mathrm{Si}(11.9)^{6}$. It has been proven that the density of accumulated carriers is proportional to the dielectric constant under identical band bending conditions ${ }^{51}$. Therefore, the strong photogating effect observed in the $\mathrm{CH}_{3} \mathrm{NH}_{3} \mathrm{PbI}_{3-x} \mathrm{Cl}_{x} /$ PEDOT:PSS vertical heterojunction is also due to the high dielectric constant of the perovskite material and the high hole mobility in the PEDOT:PSS layer.

Flexible photodetectors have recently received considerable attention because of their potential applications in several emerging areas, such as wearable electronics and artificial eyes ${ }^{52}$. As shown in Figure 4, perovskite photodetectors were fabricated on flexible polyimide (PI) substrates with a thickness of $50 \mu \mathrm{m}$. Figure $4 \mathrm{~b}$ shows the $I_{\mathrm{ph}}-V$ relationships for a representative flexible photodetector under infrared light illumination (wavelength: $\sim 895 \mathrm{~nm}$ ) at various light intensities. The device was also characterized after being bent to a radius of $7 \mathrm{~mm}$ 300 times. Figure $4 \mathrm{c}$ shows the relationships between the responsivity and bias voltage of the device under different light intensities. The maximum responsivity was found to be $\sim 8.3 \times 10^{8} \mathrm{~A} \mathrm{~W}^{-1}$, and the performance showed little change before and after the bending test. Figure $4 \mathrm{~d}$ shows the time-dependent photoresponse of the device under alternating light illumination conditions before and after 300 bending repetitions. Interestingly, the response time of the flexible device was faster than that of a device prepared on a $\mathrm{SiO}_{2} / \mathrm{Si}$ substrate under the same processing conditions, and the photocurrent could 
reach a stable value within tens of seconds. We characterized the crystalline quality of two perovskite films prepared on $\mathrm{PI}$ and $\mathrm{SiO}_{2} / \mathrm{Si}$ substrates and found that the two films showed similar diffraction patterns and peak widths (Supplementary Information and Supplementary Fig. S17). Therefore, the faster response speed cannot be attributed to a different quality of the perovskite in the flexible device. To better understand this effect, we characterized the thickness of the perovskite layer on the flexible substrate using a stylus profiler (Bruker: DektakXT, Billerica, MA, USA). We found that the thickness of the perovskite layer was only $\sim 240 \mathrm{~nm}$, thinner than the perovskite layer $(\sim 300 \mathrm{~nm})$ prepared on the $\mathrm{SiO}_{2} / \mathrm{Si}$ substrate. This difference in thickness is presumably due to the different surface morphologies and energies of the substrates. As indicated by the results shown in Supplementary Fig. S12, a thinner perovskite layer can lead to a faster photoresponse. Therefore, the faster response of the flexible device compared with the device prepared on the $\mathrm{SiO}_{2} / \mathrm{Si}$ substrate can be attributed to the thinner perovskite layer of the former.

\section{CONCLUSIONS}

In summary, we have demonstrated ultrasensitive broadband phototransistors with vertical heterojunctions based on $\mathrm{CH}_{3} \mathrm{NH}_{3} \mathrm{PbI}_{3-x} \mathrm{Cl}_{x} /$ PEDOT:PSS for the first time. Because of the high light absorption coefficient, long carrier lifetime and high dielectric constant of the perovskite, a high density of photo-generated electrons can accumulate in the perovskite layer and induce a pronounced photogating effect on the channel through the following processes. Strong light absorption in the perovskite leads to the creation of excitons or electron-hole pairs. Holes tend to diffuse into the PEDOT:PSS layer because it is energetically favorable for them to do so, leaving electrons trapped in the perovskite layer. The holes will transfer and recirculate many times in the PEDOT:PSS channel throughout their long lifetimes, giving rise to an ultrahigh gain. The devices exhibit an unprecedented responsivity of as high as $\sim 2 \times 10^{9} \mathrm{~A} \mathrm{~W}^{-1}$, a gain of $4.0 \times 10^{9}$ and a high specific detectivity of up to $\sim 1.7 \times 10^{14}$ Jones under visible light at an operating voltage of only $0.5 \mathrm{~V}$. This responsivity is several orders of magnitude higher than those achieved in previously reported perovskite photodetectors. Although the perovskite material shows weak light absorption above the absorption edge of $\sim 800 \mathrm{~nm}$, the devices still can exhibit high responsivities of close to $10^{9} \mathrm{~A} \mathrm{~W}^{-1}$ under NIR light at wavelengths of up to $1.1 \mu \mathrm{m}$ because of their extremely high gain. Moreover, high-performance devices that exhibit excellent bending stability can be assembled on flexible plastic substrates. This work opens up the possibility of the application of organolead halide perovskites in highly sensitive, low-cost and flexible photodetectors for broadband detection.

\section{CONFLICT OF INTEREST}

The authors declare no conflict of interest.

\section{AUTHOR CONTRIBUTIONS}

FY, LL and CX conceived of the experiments. CX fabricated and characterized the devices. PY and ZL assisted with experiments on device fabrication. The manuscript was written by CX and FY and was discussed, edited and approved by all authors.

\section{ACKNOWLEDGEMENTS}

This work was financially supported by the Research Grants Council (RGC) of Hong Kong, China (Project No. C4030-14G), and the Hong Kong Polytechnic University (Project No. G-YBB7, 1-BBA3, 1-ZVK1 and 1-ZVGH).
1 Gong X, Tong MH, Xia YJ, Cai WZ, Moon JS et al. High-detectivity polymer photodetectors with spectral response from $300 \mathrm{~nm}$ to $1450 \mathrm{~nm}$. Science 2009; 325: 1665-1667.

2 Arnold MS, Zimmerman JD, Renshaw CK, Xu X, Lunt RR et al. Broad spectral response using carbon nanotube/organic semiconductor/ $\mathrm{C}_{60}$ photodetectors. Nano Lett 2009; 9 : 3354-3358.

3 Konstantatos G, Sargent EH. Nanostructured materials for photon detection. Nat Nanotechnol 2010; 5: 391-400.

4 Li JH, Niu LY, Zheng ZJ, Yan F. Photosensitive graphene transistors. Adv Mater 2014; 26: 5239-5273.

5 Soci C, Zhang A, Xiang B, Dayeh SA, Aplin DPR et al. ZnO nanowire UV photodetectors with high internal gain. Nano Lett 2007; 7: 1003-1009.

6 Sze SM, Ng KK. Physics of Semiconductor Devices (3rd edn.) Hoboken: John Wiley \& Sons, Inc.; 2006.

7 Konstantatos G, Howard I, Fischer A, Hoogland S, Clifford J et al. Ultrasensitive solution-cast quantum dot photodetectors. Nature 2006; 442: 180-183.

8 Guo FW, Yang B, Yuan YB, Xiao ZG, Dong QF et al. A nanocomposite ultraviolet photodetector based on interfacial trap-controlled charge injection. Nat Nanotechnol 2012; 7: 798-802.

9 Xia FN, Mueller T, Lin YM, Valdes-Garcia A, Avouris P. Ultrafast graphene photodetector. Nat Nanotechnol 2009; 4: 839-843.

10 Liu C-H, Chang Y-C, Norris TB, Zhong ZH. Graphene photodetectors with ultrabroadband and high responsivity at room temperature. Nat Nanotechnol 2014; 9: 273-278.

11 Lopez-Sanchez O, Lembke D, Kayci M, Radenovic A, Kis A. Ultrasensitive photodetectors based on monolayer MoS 2 . Nat Nanotechnol 2013; 8: 497-501.

12 Duan XD, Wang C, Shaw JC, Cheng R, Chen Y et al. Lateral epitaxial growth of twodimensional layered semiconductor heterojunctions. Nat Nanotechnol 2014; 9: 1024-1030.

13 Konstantatos G, Badioli M, Gaudreau L, Osmond J, Bernechea M et al. Hybrid graphene-quantum dot phototransistors with ultrahigh gain. Nat Nanotechnol 2012; 7: 363-368.

14 Sun ZH, Liu ZK, Li JH, Tai GA, Lau S-P et al. Infrared photodetectors based on CVDgrown graphene and $\mathrm{PbS}$ quantum dots with ultrahigh responsivity. Adv Mater 2012; 24: 5878-5883.

15 Roy K, Padmanabhan M, Goswami S, Sai TP, Ramalingam G et al. Graphene-MoS 2 hybrid structures for multifunctional photoresponsive memory devices. Nat Nanotechnol 2013; 8: 826-830.

16 Yang Y, da Costa RC, Fuchter MJ, Campbell AJ. Circularly polarized light detection by a chiral organic semiconductor transistor. Nat Photonics 2013; 7: 634-638.

17 Baeg KJ, Binda M, Natali D, Caironi M, Noh YY. Organic light detectors: photodiodes and phototransistors. Adv Mater 2013; 25: 4267-4295.

18 Burschka J, Pellet N, Moon S-J, Humphry-Baker R, Gao P et al. Sequential deposition as a route to high-performance perovskite-sensitized solar cells. Nature 2013; 499: 316-320.

19 Liu MZ, Johnston MB, Snaith HJ. Efficient planar heterojunction perovskite solar cells by vapour deposition. Nature 2013; 501: 395-398.

$20 \mathrm{Kim}$ H-S, Lee C-R, Im J-H, Lee K-B, Moehl T et al. Lead iodide perovskite sensitized allsolid-state submicron thin film mesoscopic solar cell with efficiency exceeding $9 \%$. Sci Rep 2012; 2: 591.

21 Jeng JY, Chiang YF, Lee MH, Peng SR, Guo TF et al. $\mathrm{CH}_{3} \mathrm{NH}_{3} \mathrm{Pbl}_{3}$ perovskite/fullerene planar-heterojunction hybrid solar cells. Adv Mater 2013; 25: 3727-3732.

22 Kazim S, Nazeeruddin MK, Grätzel M, Ahmad S. Perovskite as light harvester: a game changer in photovoltaics. Angew Chemie - Int Ed 2014; 53: 2812-2824.

23 Stranks SD, Eperon GE, Grancini G, Menelaou C, Alcocer MJP et al. Electron-hole diffusion lengths exceeding 1 micrometer in an organometal trihalide perovskite absorber. Science 2013; 342: 341-344.

24 Shi D, Adinolfi V, Comin R, Yuan MJ, Alarousu E et al. Low trap-state density and long carrier diffusion in organolead trihalide perovskite single crystals. Science 2015; 347: 519-522.

25 Dong QF, Fang YJ, Shao YC, Mulligan P, Qiu J et al. Electron-hole diffusion lengths $>175 \mu \mathrm{m}$ in solution-grown $\mathrm{CH}_{3} \mathrm{NH}_{3} \mathrm{Pbl}_{3}$ single crystals. Science 2015; 347: 967-970.

26 Tan Z-K, Moghaddam RS, Lai ML, Docampo P, Higler R et al. Bright light-emitting diodes based on organometal halide perovskite. Nat Nanotechnol 2014; 9: 687-692.

27 Zhu HM, Fu YP, Meng F, Wu XX, Gong ZZ et al. Lead halide perovskite nanowire lasers with low lasing thresholds and high quality factors. Nat Mater 2015; 14: 636-642.

28 Yakunin S, Sytnyk M, Kriegner D, Shrestha S, Richter M et al. Detection of X-ray photons by solution-processed lead halide perovskites. Nat Photonics 2015; 9: 444-449.

29 Zhou HP, Chen Q, Li G, Luo S, Song T-B et al. Interface engineering of highly efficient perovskite solar cells. Science 2014; 345: 542-546.

30 Yang WS, Noh JH, Jeon NJ, Kim YC, Ryu S et al. High-performance photovoltaic perovskite layers fabricated through intramolecular exchange. Science 2015; 348: 1234-1237.

$31 \mathrm{Hu}$ X, Zhang XD, Liang L, Bao J, Li S et al. High-Performance flexible broadband photodetector based on organolead halide perovskite. Adv Funct Mater 2014; 24 : 7373-7380.

32 Xia HR, Li J, Sun WT, Peng LM. Organohalide lead perovskite based photodetectors with much enhanced performance. Chem Commun 2014; 50: 13695-13697.

33 Dou LT, Yang Y, You JB, Hong ZR, Chang W-H et al. Solution-processed hybrid perovskite photodetectors with high detectivity. Nat Commun 2014; 5: 5404. 
34 Dong R, Fang YJ, Chae J, Dai J, Xiao ZG et al. High-gain and low-driving-voltage photodetectors based on organolead triiodide perovskites. Adv Mater 2015; 27: 1912-1918.

35 Lee Y, Kwon J, Hwang E, Ra CH, Yoo WJ et al. High-performance perovskite-graphene hybrid photodetector. Adv Mater 2015; 27: 41-46.

$36 \mathrm{Li} \mathrm{F}$, Ma C, Wang H, Hu WJ, Yu WL et al. Ambipolar solution-processed hybrid perovskite phototransistors. Nat Commun 2015; 6: 8238.

37 Fang YJ, Dong QF, Shao YC, Yuan YB, Huang JS. Highly narrowband perovskite singlecrystal photodetectors enabled by surface-charge recombination. Nat Photonics 2015, 9: 679-686.

38 Lin $Q Q$, Armin A, Burn PL, Meredith P. Filterless narrowband visible photodetectors. Nat Photonics 2015; 9: 687-694.

39 Xu WT, Cho H, Kim YH, Kim YT, Wolf C et al. Organometal halide perovskite artificial synapses. Adv Mater 2016; 28: 5916-5922.

40 Grancini G, Srimath Kandada AR, Frost JM, Barker AJ, De Bastiani M et al. Role of microstructure in the electron-hole interaction of hybrid lead halide perovskites. Nat Photonics 2015; 9: 695-701.

41 Green MA, Ho-Baillie A, Snaith HJ. The emergence of perovskite solar cells. Nat Photonics 2014; 8: 506-514.

42 Shao DL, Gao J, Chow P, Sun HT, Xin GQ et al. Organic-inorganic heterointerfaces for ultrasensitive detection of ultraviolet light. Nano Lett 2015; 15: 3787-3792.

43 Liu X, Gu LL, Zhang QP, Wu JY, Long YZ et al. All-printable band-edge modulated $\mathrm{ZnO}$ nanowire photodetectors with ultra-high detectivity. Nat Commun 2014; 5: 4007.

$44 \mathrm{Kim}$ CO, Kim S, Shin DH, Kang SS, Kim JM et al. High photoresponsivity in an allgraphene p-n vertical junction photodetector. Nat Commun 2014; 5: 3249.

45 You P, Liu ZK, Tai QD, Liu SH, Yan F. Efficient semitransparent perovskite solar cells with graphene electrodes. Adv Mater 2015; 27: 3632-3638.
46 Jung HS, Park NG. Perovskite solar cells: from materials to devices. Small 2015; 11 : 10-25.

47 Zhang WJ, Chuu C-P, Huang J-K, Chen C-H, Tsai M-L et al. Ultrahigh-gain photodetectors based on atomically thin graphene- $\mathrm{MoS}_{2}$ heterostructures. Sci Rep 2014; 4: 3826.

48 Xing GC, Mathews N, Sun SY, Lim SS, Lam YM et al. Long-range balanced electron- and hole-transport lengths in organic-inorganic $\mathrm{CH}_{3} \mathrm{NH}_{3} \mathrm{Pbl}_{3}$. Science 2013; 342: 344-347.

49 deQuilettes DW, Vorpahl SM, Stranks SD, Nagaoka H, Eperon GE et al. Impact of microstructure on local carrier lifetime in perovskite solar cells. Science 2015; 348: 683-686.

$50 \mathrm{Li} \mathrm{JH}$, Sun ZH, Yan F. Solution processable low-voltage organic thin film transistors with high- $k$ relaxor ferroelectric polymer as gate insulator. Adv Mater 2012; 24: 88-93.

51 Yan F, Li JH, Mok SM. Highly photosensitive thin film transistors based on a composite of poly(3-hexylthiophene) and titania nanoparticles. J App/ Phys 2009; 106: 074501.

52 Song YM, Xie YZ, Malyarchuk V, Xiao JL, Jung I et al. Digital cameras with designs inspired by the arthropod eye. Nature 2013; 497: 95-99.

(c) (i) $(-)$ This work is licensed under a Creative Commons Attributioncc) NonCommercial-NoDerivs 4.0 International License. The images or other third party material in this article are included in the article's Creative Commons license, unless indicated otherwise in the credit line; if the material is not included under the Creative Commons license, users will need to obtain permission from the license holder to reproduce the material. To view a copy of this license, visit http:// creativecommons.org/licenses/by-nc-nd/4.0/

(C) The Author(s) 2017

Supplementary Information for this article can be found on the Light: Science \& Applications' website (http://www.nature.com/lsa). 